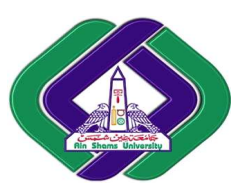

\title{
IRIS AND FINGER VEIN MULTI MODEL RECOGNITION SYSTEM BASED ON SIFT FEATURES
}

\author{
F. E. Mohammed \\ E. M. ALdaidamony \\ A. M. Raid \\ Information Science Department, Faculty of Computers and Information System, Mansoura University - Egypt \\ faresalabadi@yahoo.com \\ eman.8.2000@gmail.com \\ amriad2000@mans.edu.eg
}

\begin{abstract}
Individual identification process is a very significant process that resides a large portion of day by day usages. Identification process is appropriate in work place, private zones, banks ...etc. Individuals are rich subject having many characteristics that can be used for recognition purpose such as finger vein, iris, face ...etc. Finger vein and iris key-points are considered as one of the most talented biometric authentication techniques for its security and convenience. SIFT is new and talented technique for pattern recognition. However, some shortages exist in many related techniques, such as difficulty of feature loss, feature key extraction, and noise point introduction. In this manuscript a new method named SIFT-based iris and SIFT-based finger vein identification with normalization and enhancement is proposed for achieving better performance. In evaluation with other SIFT-based iris or SIFT-based finger vein recognition algorithms, the suggested technique can overcome the difficulties of accurate extraction of key-points and clear the noise points without feature loss. Experimental outcomes demonstrate that the normalization and improvement steps are critical for SIFT-based iris recognition and SIFT-based finger vein recognition, the recommended method can accomplish satisfactory recognition performance.
\end{abstract}

Keywords:SIFT, Iris Recognition, Finger Vein RecognitionandBiometeric Systems.

\section{Introduction}

Biometric identification systems were typically used in forensic sciences, but these days it is mostly due to national applications such as controlling physical access to facilities, controlling logical access to software, and controlling voters during elections. A key factor in fingerprint recognition systems is the fingerprint identical algorithm. No single biometric feature will gather all the conditions of every possible application. But, the popular of new patterns used in the recognition method is finger vein [1].

Finger veins are subcutaneous structures that randomly develop into a network and spread a long a finger [2] and considered to be quite unique, even in the case of matching twins and even between the different fingers of an individual proved by [3]. As a biometric identifier, finger veins have many characteristics, such a universality, individuality, stability, etc. In comparison with other biometric identifiers such as finger print, face, hand geometry and gait, finger veins express some tremendous advantages in application [4][5]. (1) Live body identification: finger veins can only be identified on a live body without fake finger attack sin finger print recognition; (2) immune to counterfeit: finger vein feature keys are interior features that are hard to forge; (3) non-contact: finger veins are not affected by 
exterior conditions. Non-invasive and contact less data capture ensures both convenience and clean lines for the users; and (4) smaller size of devices : most finger vein capturing devices are slighter in size as evaluated to palm vein based authentication devices.

Iris identification is participating a more and more significant role in many mission-critical applications recently, such as access control, personal identification, border crossing, E-passport, etc. Usually, iris identification has higher precision and consistency evaluated with other biometrics. It is highly believed that it is impossible to get two identical iris images from two persons, even if they are twins. Generally speaking, iris identification involves preprocessing, key-points extraction and matching. The preprocessing phase mainly contains iris position and normalization. After preprocessing, iris image key-points can be computed, extracted for matching.

In this manuscript, we suggest a SIFT based iris identification technique to handle the above problems, where key-points can be characterized well with improvement, enhancement and some fake key-points can be excluded with normalization. Experimental outcomes display that the recommended technique is more efficient to extract features from iris images and it can realize a higher accuracy. The rest of this manuscript is categorized as follows Sect. 2 discuses related work of biometric recognition systems, and Sect. 3 describes the recommended framework and technique based on SIFT. In Sect. 4, experiments results and analysis will be presented and in the last section conclusions and references will be given.

\section{Related work}

\subsection{Background}

Ghazvini, Met. Al. suggest a fingerprint matching technique based on genetic algorithm. Then use the local properties of minutiae. Also there is a new type of triangle descriptors are proposed, which uses these descriptors assets, the approximated value of transfer limitation for alignment and similarity between two fingerprints can be attained [6].

Prabhakar, S et. al. discussed the signal achievement portions of fingerprint and iris biometrics-two of the most generally used biometric traits. Personal recognition of people is essential to conduct many social and economic activities. [7].

N. Miura et. al. have achieved robust feature keys extraction for finger vein. They have proposed a technique of personal recognition based on finger-vein patterns. In which finger vein image is taking under infrared light contains not only the vein pattern but also unbalanced shading produced by the various width of the finger bones and muscles. The proposed technique extracts the finger-vein feature keys from the uncertain image by using line tracking that starts from various positions [8].

HimanshiBudhirajaetet.al. proposes a biometric individual verification system using a novel mixture of iris and fingerprint. For system deployment the combination is found to be useful as one needs a close up system and other needs contact. One modality is used to conquer the restrictions posed by the other.

Zhu et al. [9] introduced SIFT to iris feature key extraction for the difficulties such as incorrect localization, occlusion, and nonlinear elastic deformation. This technique used annular and normalized iris pictures without improvement for feature extraction. It can reduce iris preprocessing steps. Furthermore, due to the profits of SIFT, it can illustrate iris local properties efficiently.

However, experimental results show that feature points cannot be represented well without image enhancement. That is because many useful minute textures of iris images tend to be ignored. As a result, 
it is not very suitable to apply SIFT to get enough extreme points and stable feature vectors to represent iris images.

\subsection{SIFT}

SIFT (Scale Invariant Feature Transform) was first introduced by Lowe to get better performance in object recognition. Due to its benefits of invariance to scale, rotation and affine transformation, SIFT is soon used in many fields, especially in biometrics. The fundamental standard of SIFT is to find extreme points in scale space and filter them to get stable ones, and then local features of the images around these stable points are extracted, thus local descriptors are generated from these local key-points for matching.[10]

SIFT algorithm consists of four main stages: a) scale-space detection, b) key-point localization, c) orientation assignment and d) key-point descriptor. The first phase used difference-of-Gaussian (DOG) function to recognize possible interest points, which were invariant to scale and orientation. DOG was used instead of Gaussian to improve the computation speed [8].

$$
D(x, y, \sigma)=(G(x, y, k \sigma)-G(x, y, \sigma)) * I(x, y)=L(x, y, k \sigma)-L(x, y, \sigma)
$$

where $*$ is the convolution operator, $\mathrm{G}(\mathrm{x}, \mathrm{y}, \delta)$ is a variable-scale Gaussian, $\mathrm{I}(\mathrm{x}, \mathrm{y})$ is the input image $\mathrm{D}(\mathrm{x}, \mathrm{y}, \delta)$ is Difference of Gaussians with scale $\mathrm{k}$ times as in equation 1. In the key-point localization step, they are discarded the points with low-contrast and eliminated the edge response. According to experiments, the descriptor of SIFT that was used is $4 \times 4 \times 8=128$ dimensions.

\section{Proposed Framework}

To handle the difficulties above, we proposed a new technique which applies enhancement and normalization preprocessing to SIFT.The framework of proposed technique is presented in Fig.1.

\subsection{Iris Recognition}

Proposed framework is a multimodal biometric identification that divided into iris recognition and finger vein identification. The first phase is iris recognition that divide into seven main stages 1) Iris localization and segmentation 2) Pupil Detection 3) Normalization 4) SIFT Feature Extraction 5) SVM Feature Classifier 6) Genetic Optimization.

\subsubsection{Iris Localization Preprocessing}

Iris position is a key step for identification, and the precision of location can influence the effect of normalization and feature extraction to some extent. Circle detection algorithm is used to enhance the speed of the system. Circle detection is used because of many benefits. Circle detection algorithm has enough recognition performance and speed level. Also, it has great ability for accurately detection with high correctness although partially occluded circles. Circle detection is simpler, efficient, optimum memory consumer and low burden processing method than others. The classical location algorithms include Daugman's circular edge detector algorithm and Wildes' Hough transform algorithm. Circular edge detector has a high accuracy in location, but it is time-consuming and is easily affected by local obstruction (especially illumination affection). Although algorithm of Hough transform can eliminate the affection of noise points and discontinuous edge, the common search also needs complex computations and the algorithm cannot solve the dilemma on binaryzation threshold selection. To overcome these difficulties, an improved Hough transform algorithm is proposed by Wang et al. to locate iris images. 


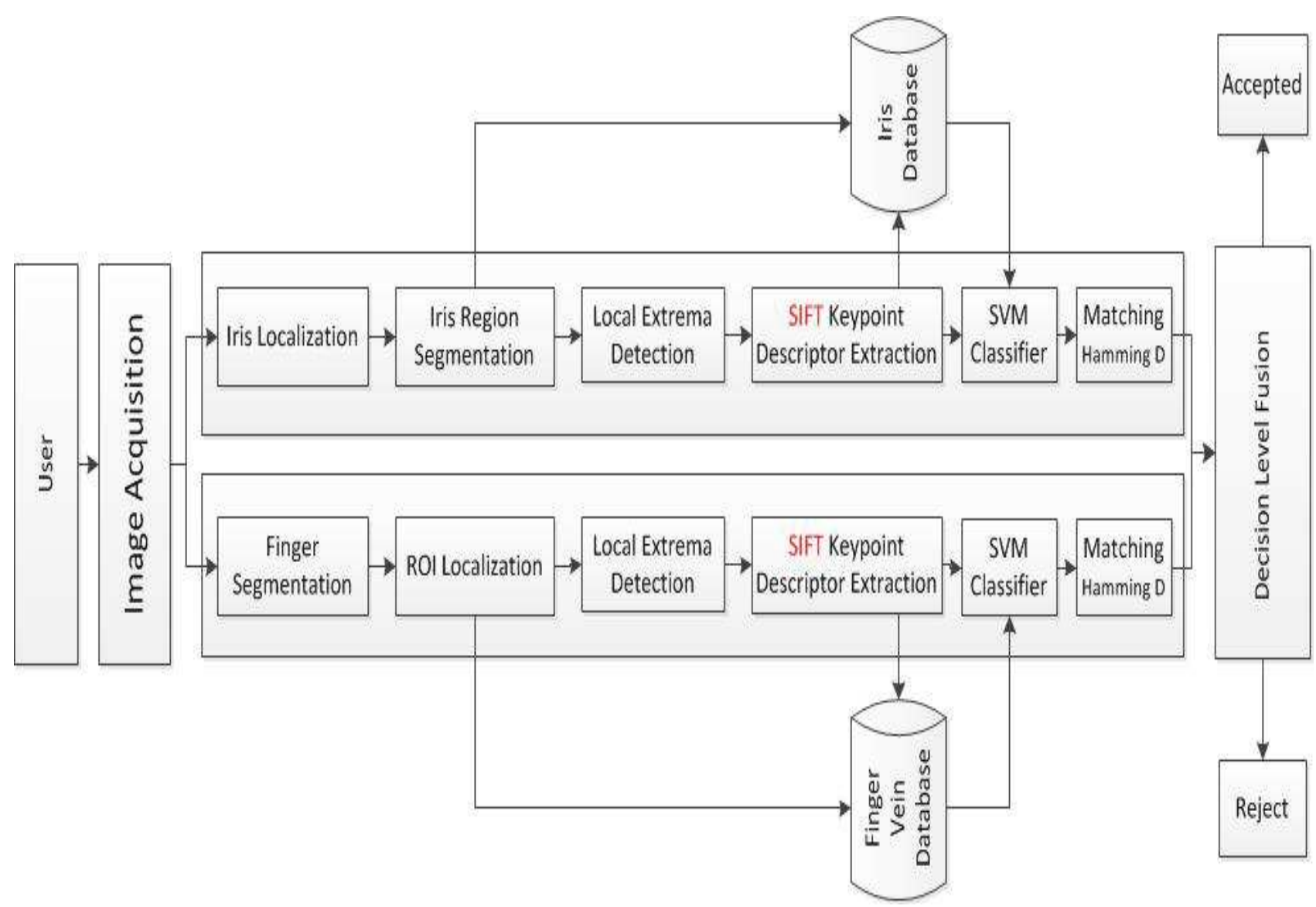

Figure.1. Proposed Framework

\subsubsection{Normalization}

Normalization can not only compensate the size variation caused by capture distance to eyes, but also overcome the influence of rotation, translation and scale to a certain extent for the following feature extraction algorithm. Experimental outcomes demonstrate that normalization is useful for enhancement in the strength on iris data sets, and features extracted with normalization are more correct compared with non-normalized ones. The traditional technique of iris normalization is to change annular iris pictures from polar coordinates to orthogonal coordinates. Fig.2 illustrates the result of four phases of iris identification which Fig. 2.a location, fig.2.b normalization, fig.2.c enhancement and fig.2.d matching. 


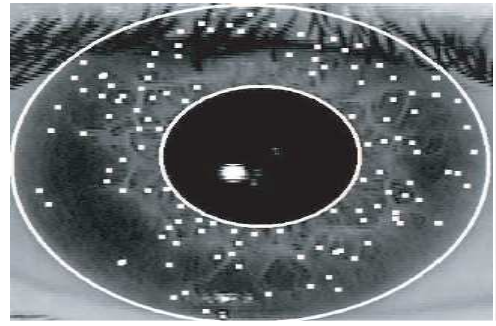

(a)

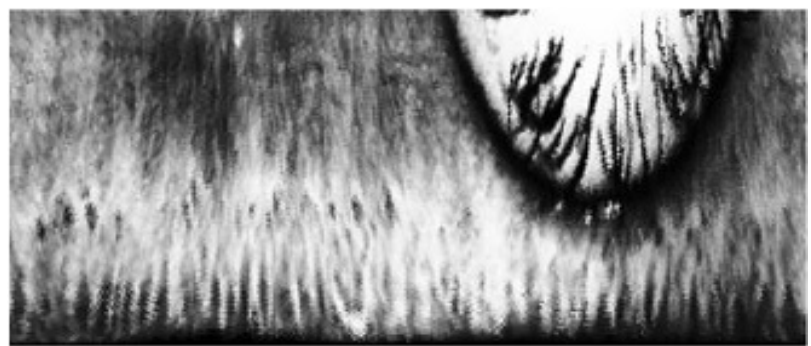

(c)

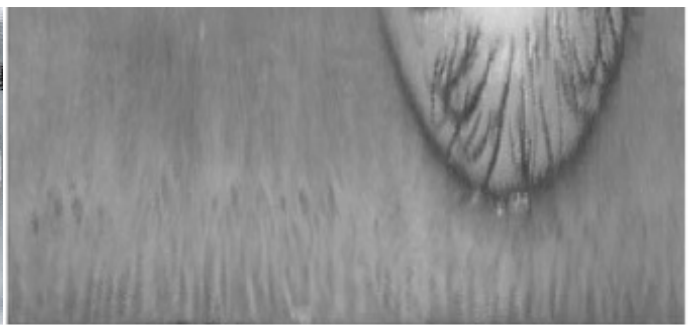

(b)

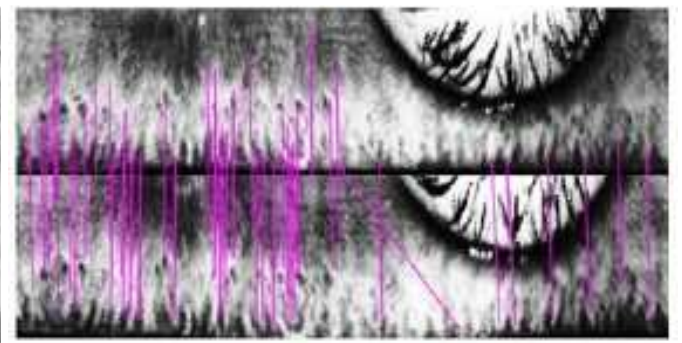

(d)

Figure.2. Four steps of iris recognition: (a) location, (b) normalization,

(c) enhancement, (d) matching

\subsection{Finger Vein Recognition}

In this part, we describe the second phase finger vein identification technique in detail . It mainly involves four steps : preprocessing, minutiae extraction, feature descriptors calculation using SIFT and minutiae matching, in which the minutiae matching involves three stages: (i)minutia pairing, (ii) false removing.

\subsubsection{Preprocessing and Minutiae Extraction}

The obtained finger pictures are low similarity and noisy with rotating and translating variations resulting from unconstrained imaging, therefore they should be initially subjected to preprocessing steps. On account of the similar preprocessing operation of two databases, in this part, we take the example of MLA database to present our preprocessing operation. It mainly refers to process the gray image gray, region of interest (ROI) extraction, image normalization, image segmentation and image thinning. Similar to[29], gray image processing is used for transforming the original 24-bit color image into an 8-bit gray image for reducing the computational complexity. ROI can be obtained according to the maximum value and minimum value of the finger contour after finger contour extraction. Then the ROI area is normalized to the equal size by using the bilinear interpolation, the size of the normalized ROI is set to be 96 X 64 . After size normalization, gray normalization is used to achieve a uniform gray distribution. For finger vein segmentation, a threshold image algorithm stands on the concave detection is utilized here. First, concave region is extracted by calculating the maximum convolution in eight directions of pixels, then threshold image is constructed and binary finger vein network is segmented, as presented in Fig. 3(b). To obtaining the structure of the finger vein network, an image thinning method proposed in [11] is utilized to obtain the skeleton of finger veins. Some finger vein skeletons are presented in Fig. 3(c). 

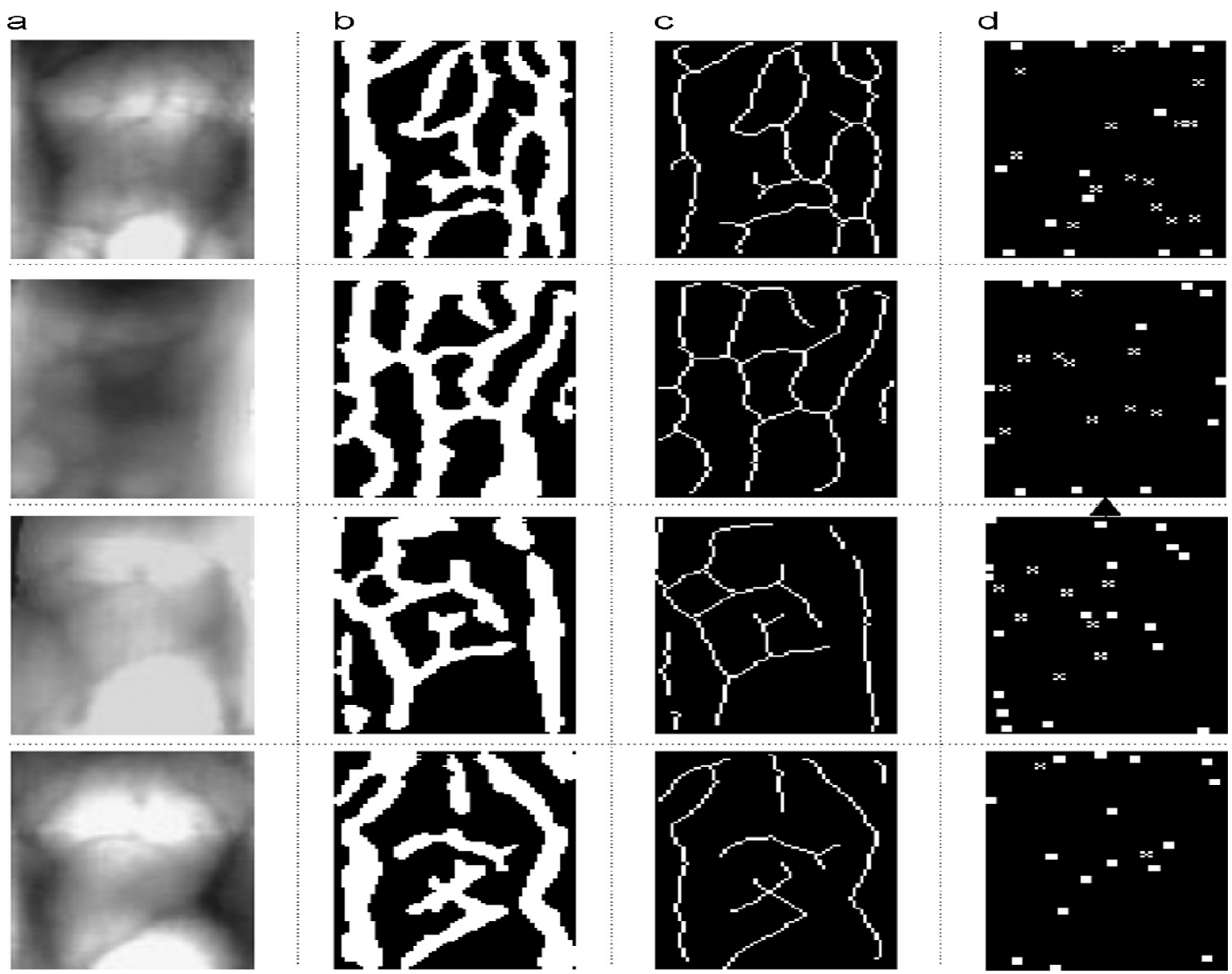

Figure.3. Finger vein images: (a) some normalized finger vein images; (b) finger vein segmentation; (c) finger vein skeletons (enhancment); and(d) minutia points

\subsection{Feature Extraction using SIFT}

The main phases of SIFT are explained as follows:

\subsubsection{Use a difference-of-Gaussian function to realize scale-space extreme detection}

The scale of an image I (x,y) can be defined as a function which can be computed by the complication of Gaussian function and image with Eq. 2:

$$
L(x, y, \delta)=G(x, y, \delta) \times I(x, y)
$$

In order to get established key-point in scale-space, Eq. 3 is given to calculate extreme in scale-space in which $\mathrm{k}$ is the constant of two neighboring scales.

$$
L(x, y, \delta)=(G(x, y, k \delta)-G(x, y, k \delta)) * I(x, y)=L(x, y, k \delta)-L(x, y, \delta)
$$

\subsubsection{Select stable key-points from extreme points}

Having been gained by the first step, unbalanced extreme points (low-contrast points and edge points) can be excluded to realize stability and anti-noise ability, and the stable key-points will be selected from 
these extreme points. For one extreme point $\mathrm{A}$ in a certain scale-space spaceD $(\mathrm{x}, \mathrm{y}, \delta)$

\subsubsection{Assign orientations to each key-point}

One key characteristic of SIFT is rotational invariance, and the perception of "relative", is utilized for reason of rotation invariance. In other vocabularies the descriptors of key-points are relative to directions, by which the rotational invariance can be recognized. Based on the scale of key-point, we prefer the Gaussian smoothing image $L$ which has the adjacent scale with the key-point. And for each point $\mathrm{L}(\mathrm{x}, \mathrm{y})$ of image $\mathrm{L}$ gradient and directions will be computed with Eq. 4:

$$
m(x, y)=\sqrt{(L(x+1, y)-L(x-1, y))^{2}+(L(x, y+1)-L(x, y-1))^{2}}
$$

Based on the center of key-points, a region is defined to form a direction histogram by computing gradient of all points in it. The directions information of the key-point can be computed by the histogram to get the feature, and the feature may have more than one direction. Besides, the evaluated Gauss gradients of key-points' neighbor can improve the stability of the features.

\subsubsection{Generate local key-point descriptors}

The previous operations have assigned orientations to each key-point, and then the local key-point descriptors can be generated. Each defined region of key-point will be separated into several subregions, each having 8 directions. In our experiments, a region with $16 \mathrm{X} 16$ pixels can be separated into 16 sub-regions. Each sub-region includes $4 \times 4$ pixels and generates a descriptor with 8 directions, thus feature vector with $16 \times 8$ dimensions of each key-point can be generated.

\subsubsection{Enhancement}

The problems caused by different locations of light source after normalization, such as low-contrast and non-uniform illumination, will influence the following recognition process. The enhancement step is used to describe features of iris or vein images better. Many related enhancement algorithms were proposed such as histogram equalization, entropy enhancement, background subtraction, and SVM enhancement. In this manuscript, histogram equalization is utilized, because it is practicable and low time consumption. The purpose of histogram equalization is to get the same amount of pixels in each grey level by point operations. The current pixel value of is $G_{A}$ and the $G_{B}$ is the value of corresponding pixel after transformation, and $\mathrm{G}_{\mathrm{B}}$ can be computed with Eq. 5, where MXN is the resolution of the image. $\mathrm{H}_{\mathrm{i}}$ is the total number of pixels in the ith grey level and Gmax refers to the maximal grey level.

$$
G_{B}=\frac{G_{\max }}{M \times N} \sum_{i=0}^{G_{A}} H_{i}
$$

Key-points can be characterized more correctly and more extreme points can be extracted with enhancement, which conquer the limitation of minutiae feature loss and is necessary for matching. The result of enhancement is presented in Fig.2.c.

\subsection{Matching}

In this stage, key-points are extracted from two iris pictures to be matched with SIFT algorithm respectively. Based on key-points extracted from each image, the same key-points are selected as 
matching pairs and the number of matching pairs is used to determine the similarity of these two iris or vein images by using hamming distance and result of matching shows in fig. 4 .

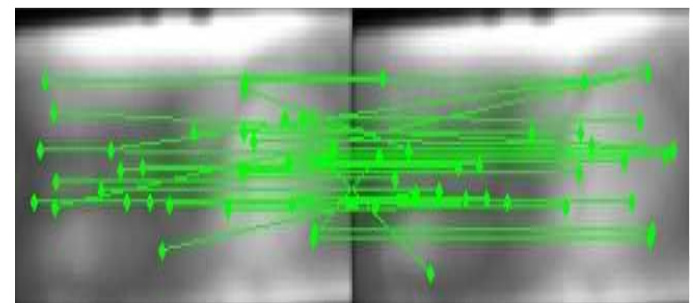

(a)

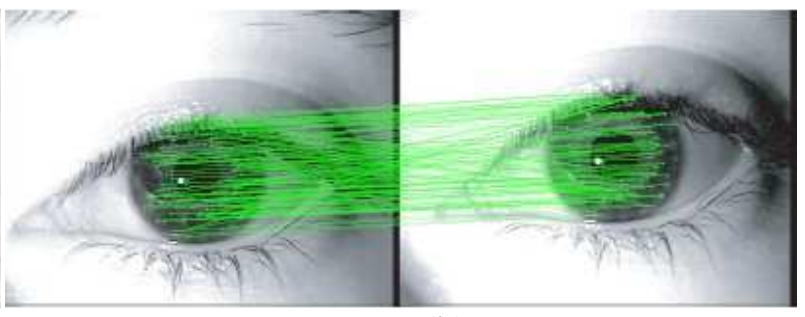

(b)

Figure.4. Result of matching process: (a) two images of finger vein (b) two images of iris

\subsection{Fusion}

The two normalized similarity scores $\mathrm{N}_{\text {Iris }}$ and $\mathrm{N}_{\text {Finger }}$ are fused linearly using sum rule as in equation6

$$
\mathrm{MS}=\alpha^{*} \mathrm{~N}_{\text {Iris }}+\beta^{*} \mathrm{~N}_{\text {vein }}
$$

\section{Experimental results and analysis}

\subsection{Engine and Database}

Experiments are done out on windows 7, processor core i7 and RAM 8 GB to gain high accuracy and performance. In this manuscript, we choose the CASIA-v1 iris database and MLA database for finger vein dataset to evaluate the results with different methods of different iris identification techniques based on SIFT. The dataset consists of 108 modules and each module has 7 pictures captured in two sessions. All images are stored in BMP format with resolution 320X280.

\subsection{Experimental Discussion}

The performance indicators computed in this experiment are FMR and FMR. These measures are helpful to describe the precision of finger vein based systems, which are often operated far from the EER point using thresholds which reduce FMR at the cost of higher FNMR. False match rate (FMR): The probability of illegal inputs which are wrongly accepted.

$$
\text { FAR }(\text { or FMR })=\frac{\text { Number of Falsely accepted images }}{\text { Total number of persons out the databese }}
$$

False non-match rate (FNMR): the probability of legal inputs which are wrongly rejected.

$$
\text { FRR }(\text { or FNMR })=\frac{\text { Number of Falsely rejected images }}{\text { Total number of persons in the databese }}
$$

We design experiment to verify the efficiency of our method. In experiment, four preprocessing results will becompared, namely annular without enhancement, annularwith enhancement, normalization without enhancement andnormalization with enhancement. Experimental resultsindicate that proposed method can achieve a better performance. All these experiments use the sameparameters, which ensures the matching process only to beaffected by preprocess. Figure 5 shows four receiver operatingcharacteristic ROC (Receiver operating characteristics) curves and Table 1 shows the equalerror rate (EERs) are 23, 12, 22 and $5 \%$ forannular without enhancement, annular with 
enhancement,normalization without enhancement and normalization withenhancement, respectively. Table 2 shows the compartive study in FAR, FRR and accuracy between single-model biomertric system (using Iris or Finger vein only) and the Multi-modelbiomertic system with feature level fusion (using Iris and finger vein together). Table 2 and Figure 6shows that the multi-model biometeric system increase accuray and reduce FAR and FRR.

\begin{tabular}{|ll|}
\hline Method & ERR \% \\
\hline Annular without enhancement & 23 \\
\hline Annular with enhancement & 12 \\
\hline Normalization without enhancement & 22 \\
\hline Normalization with enhancement & 5 \\
\hline
\end{tabular}

Table.1.EERs of four different preprocessing techniques of Iris Recognition

\begin{tabular}{|l|c|c|c|}
\hline & FAR\% & FRR\% & Accuracy\% \\
\hline Iris & $5 \%$ & $7 \%$ & $96 \%$ \\
\hline Finger vein & $10 \%$ & $8 \%$ & $94 \%$ \\
\hline $\begin{array}{l}\text { Iris+finger } \\
\text { vein }\end{array}$ & $2 \%$ & $3 \%$ & $98 \%$ \\
\hline
\end{tabular}

Table.2.Experimental Results of single and multi model biometric system

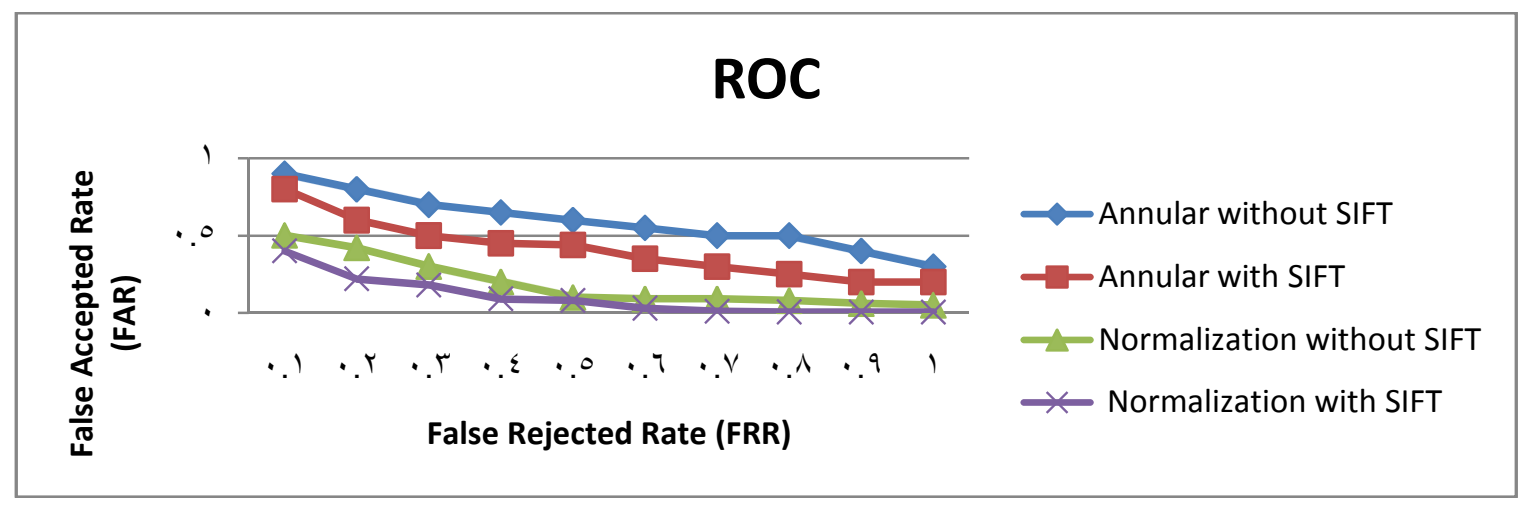

Figure.5.ROC curves of four methods of Proposed Iris Recognition as Unimodel

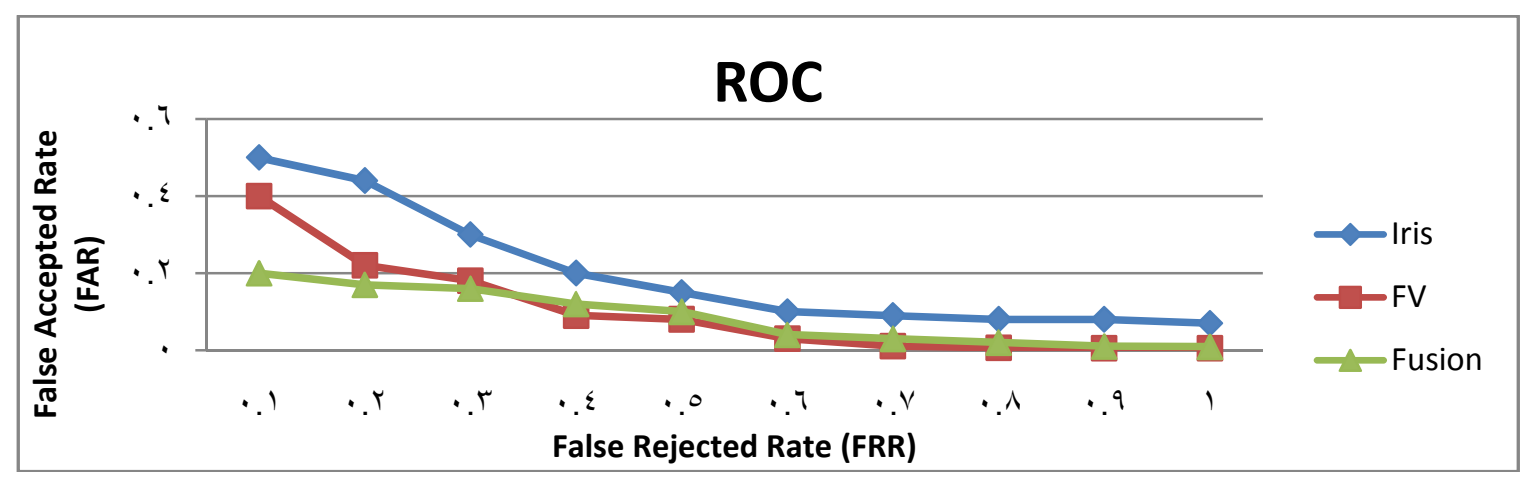

Figure.6. ROC curves of three methods of Proposed Framework Recognition System as Multimodel 


\section{Conclusion}

Biometrics is a very talented technology, challenges are slowing its development and deployment. Finger vein and Iris images are selected due to their unique physiological traits. The proposed multimodal biometric recognition system is considered a robust mixture of finger vein and iris keypoints that have matching score level with accuracy of $98 \%$ with FAR and FRR of $2 \%$ and $3 \%$. The recommended system is a gentle secure and robust stable identification and recognition system. The recommended system is a new combination to the biometric research that can be extend and enhanced during times.

\section{References}

1. RAVI. J, K. B. RAJA, VENUGOPAL. K. R," FINGERPRINT RECOGNITION USING MINUTIA SCORE MATCHING", Ravi.J. et al /IJEST Vol.1(2), 35-42, (2009).

2. M. Xue,Q.Sun,Vasculaturedevelopmentinembryosanditsregulatory mechanisms, Chin.J.Comp.Med.13(1)45-49, (2003).

3. T.Yanagawa,S.Aoki,T.Ohyama,Human finger vein images are diverseandits patterns areusefulforpersonalidentification, MHFPreprintSeries,Facultyof Mathematics, KyushuUniversity,Fukuoka-JAPAN,April (2007).

4. J.Yang,Y.Shi,J.Yang,L.Jiang,Anovel finger-veinrecognitionmethodwith feature combination,in:IEEEInternationalConferenceon Image Processing,ICIP,pp.2709-2712 ， Cairo, (2009).

5. J. Tang,X.T.Zhen, context",PatternRecognit.47(3)1469-1484, (2014).

L.Shao,"Robustpointpatternmatchingbasedonspectral

6. Ghazvini, M, Mohammadi, K, Sufikarimi, H. "Fingerprint Matching Using Genetic Algorithm andTriangle Descriptors". In Proceedings of the 19th Iranian Conference on Electrical Engineering,Tehran, Iran, pp. 1-6, (17-19 May 2011).

7. Hoyle, K. "Minutiae Triplet-Based Features with Extended Ridge Information for Determining Sufficiency in Fingerprints". Master Thesis, Virginia Polytechnic Institute and State University, Burruss Hall Blacksburg, VA, USA, (2011).

8. Prabhakar, S., Jain, A.K, Ivanisov, "A. Biometric recognition: Sensor characteristics and image quality". IEEE Instrum. Meas. Mag., 14, 10-16, (2011).

9. Zhu R, Wu R, Yang J, "Iris recognition based on key-point matching". In: International symposium on communicationsand information technologies, Bangkok, pp 451-454, (2009)

10. Lowe DG, "Distinctive image features from scale-invariantkeypoints". Int J Comput Vis 60(2):91$110,(2004)$

11. C.B. Yu,Y.Z.Cui, L.Zhang, H.F.Qin,"Fingerveinimagerecognitionwithminutiaefeaturematching",Interdiscip.Sci.: Comput. LifeSci.1(4)280-289, (2009). 